Revue de droit comparé du travail et de la sécurité sociale

3 | 2018

La participation des travailleurs dans la grande entreprise privée et publique

\title{
Le système allemand de participation des travailleurs au niveau de l'entreprise
}

\section{Wolfgang Däubler}

\section{(2) OpenEdition}

\section{Journals}

Édition électronique

URL : https://journals.openedition.org/rdctss/1891

DOI : $10.4000 /$ rdctss. 1891

ISSN : 2262-9815

Éditeur

Centre de droit comparé du travail et de la sécurité sociale

Édition imprimée

Date de publication : 1 novembre 2018

Pagination : $70-81$

ISSN : 2117-4350

Référence électronique

Wolfgang Däubler, «Le système allemand de participation des travailleurs au niveau de l'entreprise », Revue de droit comparé du travail et de la sécurité sociale [En ligne], 3 | 2018, mis en ligne le 01 novembre 2021, consulté le 13 novembre 2021. URL : http://journals.openedition.org/rdctss/1891 ; DOI : https:// doi.org/10.4000/rdctss. 1891

\section{(c) (i) $\ominus$}

Revue de droit comparé du travail et de la sécurité sociale est mise à disposition selon les termes de la Licence Creative Commons Attribution - Pas d'Utilisation Commerciale - Pas de Modification 4.0 International. 


\title{
LE SYSTÈME ALLEMAND DE PARTICIPATION DES TRAVAILLEURS AU NIVEAU DE L'ENTREPRISE
}

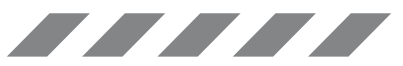

\begin{abstract}
The German codetermination exists in three forms. It has to adapt itself to the framework of company law. Workers' representatives in the supervisory board have the same rights and obligations as the representatives of the owners; they are bound by the interest of the enterprise. They are in a minority position. Their only "weapon" is the argument; having no experts there is no balance, however, with the top management and the representatives of capital. But there is a second channel: enterprise decisions may be an object of collective bargaining.
\end{abstract}

KEYWORDS : Codetermination, Imbalance Between Workers and Capital in the Supervisory Board, History of Codetermination, Supervisory Board, Codetermination and Company Law, Codetermination and Collective Bargaining.

\section{RÉSUMÉ}

La cogestion allemande existe en trois formes différentes. Elle s'insère dans le cadre du droit de sociétés. Les représentants des travailleurs au conseil de surveillance ont les mêmes droits et les mêmes obligations que les représentants du capital; ils sont donc liés par l'intérêt de l'entreprise. En même temps, ils sont dans une situation minoritaire. Leur seul instrument de "pression" est l'argument, mais faute d'experts un équilibre avec la direction et les représentants du capital n'existe pas. Mais il y a une deuxième voie: des décisions d'entreprise peuvent être l'objet de négociations collectives.

MOTS CLÉS : Cogestion, disparité entre travail et capital dans les conseils de surveillance, histoire de la cogestion, conseil de surveillance, cogestion et droit de sociétés, cogestion et négociation collective. 
nfluencer les décisions prises par l'employeur est une des plus vieilles revendications du mouvement ouvrier. Les grèves de protestation au XIXème siècle ont eu pour but surtout d'obtenir un salaire décent pour vivre et des règles de protection de la santé. Après la Première Guerre Mondiale les syndicats ont été reconnus et purent poursuivre ces buts d'une façon plus systématique. Dans son article 165, la Constitution Allemande de 1919 ("Constitution de Weimar ») est allée plus loin en donnant aux ouvriers et aux employés le droit de participer comme partenaires égaux au développement des forces productives ${ }^{1}$. La Constitution ne connaît donc pas de restriction de la compétence des syndicats en matière de salaires et de conditions de travail. Cette décision a été inspirée par le mouvement des conseils ("Rätebewegung ») qui demandait une démocratisation universelle de la société et était à la base de la révolution de $1918^{2}$; elle était soutenue aussi par des catholiques de gauche se rapportant à l'encyclique (Rerum Novarum) du pape Léon XIII.

L'article 165 de la Constitution de Weimar exigeait une intervention du législateur. En 1920, celui-ci a promulgué la loi sur les conseils d'établissement, précurseurs des conseils d'aujourd'hui. Les conseils avaient des droits de codétermination sur certaines conditions de travail. En 1922 le Parlement a ajouté une règle selon laquelle de conseil avait le droit de nommer un délégué au conseil de surveillance de l'entreprise (et deux délégués, quand le conseil de surveillance avait plus de trois membres). Les délégués devaient représenter les intérêts des travailleurs et soutenir leurs revendications sur l'organisation de l'établissement. Ils avaient un droit de vote sur toutes les questions décidées par le conseil de surveillance. En comparaison avec les souhaits nés du mouvement des conseils c'était un élément fort modeste de cogestion, largement critiqué par ceux qui étaient restés fidèles aux idées originelles de ce mouvement. Leur importance pratique n'a jamais attiré l'intérêt des sociologues; on ne trouve pas d'études sur leurs activités.

En 1947, les forces britanniques d'occupation ont repris l'idée de cette forme de cogestion. Ils ont introduit des conseils de surveillance à composition paritaire dans l'industrie du charbon et de l'acier. Leur structure et leurs fonctions seront analysées ci-après ${ }^{3}$. Les employeurs ne s'y sont pas opposés car ils considéraient ce modèle comme une alternative à la socialisation des entreprises qui, à l'époque, était à l'ordre du jour 4 .

1 Texte original: «Arbeiter und Angestellte sind dazu berufen, gleichberechtigt an der gesamten wirtschaftlichen Entwicklung der produktiven Kräfte mitzuwirken ».

2 Cf. von Oertzen, Betriebsräte in der November-Revolution, 2. Aufl., Berlin und Godesberg 1976.

3 Sous II 3.

4 Références dans W. Däubler, Das Arbeitsrecht 1, 16 e édition 2006, p. 768 ss. 
Dés 1946, les quatre puissances d'occupation, réunies dans le Conseil de contrôle allié ont promulgué une loi sur les conseils d'établissements valable dans toutes les zones d'occupation. Le conseil d'établissement pouvait définir lui-même sa compétence. Quand il voulait l'établir juridiquement, il pouvait conclure une convention avec l'employeur. En cas de désaccord, le recours à la grève était possible ${ }^{5}$. L'influence du conseil n'était donc pas forcément restreinte aux problèmes de salaire et de conditions de travail.

Dans les années suivantes, ces règles furent intégrées dans le droit allemand et modifiées d'une façon considérable ${ }^{6}$. Pour mieux comprendre les différents modèles qui ont été établis, il faut insister sur le modèle dualiste de direction des entreprises retenu en Allemagne.

La participation des travailleurs au niveau de l'entreprise est organisée par le droit des sociétés. En Allemagne, l'organisation sociétaire est fondée sur un modèle dualiste. Au lieu d'un conseil administratif unique, il existe une séparation entre le directoire et le conseil de surveillance.

Le directoire gère l'entreprise en prenant et en exécutant des décisions. Dans le cadre d'une société anonyme (Aktiengesellschaft, AG), il a pour appellation "Vorstand " et son indépendance est garantie par la loi. Dans le cadre d'une société à responsabilité limitée (Gesellschaft mit beschränkter Haftung, GmbH), il a pour nomination "Geschäftsführung"; il peut être soumis aux directives des associés mais ce n'est pas forcément le cas.

À côté du «Vorstand » et de la «Geschäftsführung » il existe le conseil de surveillance "Aufsichtsrat »). II a principalement pour rôle de nommer les membres du comité de direction et d'en contrôler l'action. En outre, le bilan annuel de l'entreprise requiert son consentement. Les statuts de la société peuvent en outre prévoir que certaines décisions du directoire doivent, pour être valables, être soumises à son approbation.

Les autres formes de sociétés - comme la société en nom collectif, la société en commandite simple, la société civile professionelle - et l'entrepreneur individuel sont beaucoup plus libres dans la création de leurs organes directeurs; ils échappent tous à la cogestion des travailleurs.

Hormis une exception, la participation des travailleurs se réalise uniquement dans le cadre du conseil de surveillance: la loi leur permet d'élire une partie des membres de ce conseil.

5 Pour plus de détails, voir E. Schmidt, Die verhinderte Neuordnung 1945 - 52, 2. Aufl. 1971, p. 90 ss.; Kleßmann/Friedemann, Streiks und Hungermärsche im Ruhrgebiet 1946 - 1948, 1977, p. 35 ss.

6 Sur les éléments alternatifs introduits par les forces d'occupation et leur absorption dans les années suivantes voir WW. Däubler, «Alternative Ansätze im Arbeitsrecht », in Dreßler/Negt/Wolter (dir.), Jenseits falscher Sachzwänge. Detlef Hensche zum 60. Geburtstag, 1998, p. 56 - 74. 


\section{I - LES TROIS MODÈLES DE COGESTION}

\section{A - Election PAR LeS tRAVAILleURS D'UN TIERS DES MEMBRES DU CONSEIL}

Dans les sociétés anonymes et dans les $\mathrm{GmbH}$ qui emploient entre 500 et 2000 travailleurs salariés, un tiers des membres du conseil de surveillance sont désignés par les travailleurs. La loi s'applique aussi aux sociétés en commandite par actions, aux sociétés coopératives (" eingetragene Genossenschaften ») et aux sociétés d'assurance mutuelle ( Versicherungsverein auf Gegenseitigkeit») qui remplissent la condition d'effectif précitée. Les représentants des travailleurs sont élus au suffrage direct. S'il y a seulement une liste, les candidats sont élus au scrutin majoritaire. S'il y a plusieurs listes, un scrutin proportionnel est retenu.

Le statut de la société fixe le nombre total des sièges au conseil de surveillance attribués aux représentants des travailleurs. La loi prévoit uniquement que ce nombre doit être au moins égal au tiers. Si le conseil comporte trois membres, il doit y avoir un représentant des travailleurs, s'il a six membres il y en a deux. Si le conseil a neuf membres, les travailleurs ont droit à trois sièges. L'une de ces trois personnes peut être un syndicaliste qui ne soit pas un salarié de l'entreprise.

Si l'entreprise appartient à un groupe, il faut distinguer. S'il existe un contrat entre la société-mère et la filiale, qui soumet celle-ci aux directives de la société-mère, les travailleurs prennent part aux élections du conseil de surveillance de la société-mère. Si un tel contrat n'existe pas, les sociétés sonst considérées comme des unités indépendantes même si la société-mère possède $100 \%$ de la filiale.

Ce modèle a été introduit en 1952 pour toutes les entreprises employant plus de 500 salariés. Les règles se trouvent aujourd'hui dans le «Drittelbeteiligungsgesetz ${ }^{7}$. Le nombre exact des entreprises qui pratiquent ce modèle est inconnu; certaines estimations mentionnent 1500 à 2000 entreprises.

7 Gesetz über die Drittelbeteiligung der Arbeitnehmer im Aufsichtsrat vom 18.Mai 2004, BGBI I S. 974. 


\section{B - Election PAR LeS tRAVAILleuRs de LA MOITIÉ DES MEMBRES DU CONSEIL}

En 1976, le législateur est intervenu pour établir dans le "Mitbestimmungsgesetz " un modèle dit paritaire pour les $A G$ et les $\mathrm{GmbH}$ qui emploient plus de 2000 salariés. Les sociétés en commandite par actions et les sociétés coopératives sont incluses elles aussi, mais la loi ne s'applique pas aux sociétés d'assurance mutuelle.

Si le conseil de surveillance comprend 12, 16 ou 20 membres, le nombre de représentants des travailleurs dépend du nombre de salariés ${ }^{9}$. La moitié est élue par les travailleurs. Dans les entreprises entre 2001 et 8000 salariés une élection au suffrage direct a lieu. Dans les entreprises employant plus de 8000 salariés, les travailleurs élisent de grands électeurs qui à leur tour élisent les membres du conseil de surveillance.

Dans les groupes d'entreprises, les travailleurs de l'ensemble des filiales participent à l'élection du conseil auprès de la société-mère. Le législateur ne fait pas de distinction entre des groupes basés sur un contrat et les groupes de fait : il suffit qu'une société soit subordonnée aux directives d'une autre. Si la filiale emploie plus de 2000 salariés, elle disposera de son propre conseil de surveillance avec une composition paritaire entre représentants du capital et travailleurs.

La parité entre travail et capital a ses particularités. La loi prévoit qu'un représentant des cadres dirigeants (" leitende Angestellte ») fait partie de la représentation des travailleurs. Dans de nombreux cas, ce représentant sera systématiquement du même avis que le côté du capital, si bien que la majorité sera clairement de ce côté. Si dans un cas concret la situation est différente et le résultat du vote est par exemple 8 à 8 ou 10 à 10, il y a obligatoirement un deuxième tour. Lors de ce deuxième tour, le président du conseil dispose de deux voix. C'est donc lui qui a le pouvoir définitif de décision. Le président est élu à une majorité des deux tiers des membres du conseil. Si cette majorité n'est pas atteinte, le côté du capital désigne le président et le côté travail son suppléant. Dans la pratique, le président est toujours un représentant du capital.

Ce modèle dit paritaire de 1976 s'applique approximativement à 700 entreprises.

\section{C - LE MODÈLE DES ENTREPRISES DU CHARBON ET DE L'ACIER}

Le troisième modèle s'applique uniquement aux entreprises du charbon et de l'acier. Il a été introduit par les forces d'occupation britannique qui ont voulu démocratiser les entreprises qui, à cette époque, étaient les plus importantes. II s'agissait, de manière moins avouée, d'affaiblir des concurrents potentiels. Après la fondation de la République Fédérale en 1949, le gouvernement Adenauer a voulu étendre la loi sur les sociétés par action de 1937 et la loi sur les $\mathrm{GmbH}$ de 1892 à ces entreprises, ce qui aurait aboli toute forme de cogestion. En réaction, les syndicats ont organisé un référendum parmi les travailleurs des entreprises du charbon et de l'acier en demandant s'ils étaient prêts à faire grève pour défendre la cogestion. $92,8 \%$ des mineurs et $95,87 \%$ des ouvriers métallurgistes ont répondu positivement. Devant ces résultats sans équivoque, le gouvernement évita la confrontation et adopta le modèle existant en droit allemand en procédant à des

8 Gesetz über die Mitbestimmung der Arbeitnehmer vom 4. Mai 1976, BGBI I S. 1153.

9 Détails dans l'article 7 de la « Mitbestimmungsgesetz ». 
modifications mineures. C'est le "Montanmitbestimmungsgesetz » de 1951 qui règle encore de nos jours la cogestion dans ce secteur ${ }^{10}$.

Le conseil de surveillance d'une entreprise de ce secteur est réellement de manière paritaire. Cinq membres sont élus par l'assemblée des actionnaires ou des associés, cinq membres sont élus par les conseils d'établissement. Ces dix représentants doivent se mettre d'accord sur une " onzième personne », normalement un ancien maire, un politicien ou un expert considéré comme neutre. En cas de désaccord, il existe une procédure de conciliation. En dernier lieu, l'assemblée des actionnaires et des associés peut trancher. Mais une telle hypothèse n'est jamais survenue au cours des 65 ans d'existence de ce système.

Le conseil ainsi composé doit élire les membres du comité de direction. La loi prévoit un directeur du travail ("Arbeitsdirektor ») qui ne peut pas être élu sans le soutien de la majorité des représentants des travailleurs. Au sein du comité de direction, il est responsable de toutes les affaires sociales et personnelles. Théoriquement, on pourrait imaginer une composition paritaire du comité de direction - deux directeurs du côté capital, deux directeurs syndicalistes et une personne "neutre " qui pourrait jouer le rôle d'un médiateur en cas de conflits. Mais c'est une idée purement fictive qui ne fut jamais partagée par les syndicats. Dès le début, un arrangement tacite a été trouvé : les représentants des travailleurs élisent le directeur du travail, les représentants du capital élisent tous les autres directeurs. Les raisons n'ont pas été discutées en public. Une composition paritaire du comité de direction aurait entraîné la responsabilité des syndicats de la politique de l'entreprise, même pour des licenciements. De plus, il aurait été difficile de trouver un nombre suffisant de cadres dirigeants loyaux envers les travailleurs.

\section{II - COGESTION ET SYNDICATS}

Dans le modèle de 1952, la présence du syndicat au conseil de surveillance dépend des stipulations statutaires de la société qui déterminent la composition du conseil de surveillance. Si le conseil a au moins 9 membres, les syndicats peuvent prendre l'initiative afin qu'un des représentants des travailleurs soit une personne venant de l'extérieur, par exemple un collaborateur du syndicat ou un expert. La décision revient aux travailleurs.

Dans le modèle de 1976, la loi énonce des règles précises. Dans les entreprises dont le conseil comporte 16 ou 18 membres, les syndicats peuvent proposer des candidats pour deux sièges. Dans les conseils avec 20 membres ils peuvent en présenter trois. Ce sont normalement des collaborateurs du syndicat, parfois des experts tels des professeurs d'université. Mais c'est uniquement une proposition, les personnes proposées devant être élues par les salariés travaillant dans l'entreprise. S'il y a plus d'un syndicat dans l'entreprise, chacun peut présenter une liste, ce qui impose une élection à la proportionnelle.

10 Gesetz über die Mitbestimmung der Arbeitnehmer in den Aufsichtsräten und Vorständen der Unternehmen des Bergbaus und der Eisen und Stahl erzeugenden Industrie (10 avril 1951), Bundesgesetzblatt I, p. 347. 
Dans la version originale du modèle de 1951 (charbon et acier), le syndicat pouvait nommer deux membres (parmi les cinq du côté des travailleurs). Actuellement la situation est plus complexe. L'assemblée des conseils d'établissement peut proposer deux candidats. En droit, l'organisation syndicale la plus importante peut cependant s'opposer à ces nominations; en cas de désaccord définitif, c'est le ministre fédéral du travail qui décide. En pratique, il y a des «sondages » préalables, si bien qu'une telle procédure n'a jamais été utilisée. Les trois autres membres du côté travail sont proposés par l'organisation syndicale; un d'entre eux ne doit pas être un collaborateur syndical ou un travailleur salarié de l'entreprise. Les candidats doivent être élus par l'assemblée des conseils. L'assemblée générale des actionnaires ou des associés garde le droit d'élire tous les membres du conseil de surveillance, mais elle est liée par l'élection faite par l'assemblée des conseils. Ce principe a toujours été respecté ; les conséquences d'un refus d'élection d'un représentant des travailleurs sont de ce fait inconnues.

Comment justifier la présence syndicale dans les conseils de surveillance? L'argument traditionnel veut que la représentation des intérêts de tous les travailleurs freine les tentations de prise en compte égoïste des intérêts de l'entreprise ou de ses salariés. Les syndicats doivent donc représenter l'intérêt général du mouvement ouvrier; en pratique, cela pose toutefois des problèmes considérables.

\section{A - LeS EFFETS PRATIQUes de LA COGESTION}

Le modèle de 1952 et celui de 1976 mettent les représentants des travailleurs dans une position minoritaire. Ils ne sont pas en capacité d'exiger la tenue d'un vote au sein du conseil de surveillance. Leur rôle se limite à formuler des arguments. Les divergences du côté des représentants du capital n'existent pratiquement pas; les représentants du capital votent toujours dans le même sens. S'il y a un désaccord interne entre eux, ils trouvent toujours un compromis pour ne pas s'en remettre à la décision des travailleurs.

Tous les membres du conseil de surveillance ont les mêmes droits et obligations. II importe de regarder ces éléments de plus près. Chaque membre a un droit à l'information sur toutes les affaires concernant l'entreprise. C'est un droit individuel qui peut être exercé sans l'appui des autres membres. L'information doit être donnée par le comité de direction. Elle est diffusée automatiquement au sein de l'ensemble du conseil de surveillance afin d'assurer un niveau homogène d'informations. En pratique, les représentants des travailleurs reçoivent des informations à chaque réunion du conseil de surveillance qui ont, en principe, lieu 4 fois par an. La tâche des représentants syndicaux consiste surtout à poser des questions un peu désagréables pour le directoire. Toutefois, les membres du conseil qui sont en même temps des salariés de l'entreprise hésitent souvent à poser des questions qui peuvent être mal vues par le comité de direction ou par un des directeurs.

Dans beaucoup de firmes le conseil met en place des commissions qui sont spécialisées par exemple en matière de questions financières, de vente des produits ou de fixation du salaire des membres du comité de direction. Les travailleurs sont représentés dans ces commissions mais ils demeurent souvent en minorité numérique. De plus, la commission appelée à se prononcer sur les salaires des directeurs ne comporte pas de représentants syndicaux. Les représentants des salariés de l'entreprise sont priés de ne pas déléguer de représentants syndicaux par crainte d'indiscrétions. C'était par exemple une pratique générale dans les banques. Aujourd'hui, le problème a perdu de son importance parce 
que le législateur a prévu après la crise de 2008 que les salaires des directeurs soient discutés en session plénière par le conseil de surveillance.

Si les relations personnelles entre les représentants des travailleurs et les membres du directoire sont bonnes, le côté travail peut obtenir des informations de manière informelle qui sont souvent plus importantes que celles données dans les réunions. Mais cela a un coût élevé : il faut une loyauté très claire. Si un représentant fait des choses considérées comme « désagréables » ou « inacceptables » (telles des déclarations à la presse critiquant la politique de l'entreprise ou l'organisation d'une grève), ce sera la fin de la collaboration tacite avec tous les représentants des travailleurs.

Tous les membres du conseil de surveillance sont liés par l'intérêt de l'entreprise: toute l'action d'un conseiller doit être orientée en fonction de cet objectif. Cette notion très large n'a été jamais définie par la jurisprudence. En doctrine, il y a une position pluraliste qui donne le même rang aux intérêts des travailleurs et à ceux du capital, tous deux se rattachant à la notion de bien public. Une autre position met l'accent sur le bon fonctionnement de l'entreprise et sa rentabilité qui serviraient au mieux les intérêts des travailleurs et le bien public ${ }^{11}$. Une conséquence concrète réside dans l'obligation de discrétion, de ne pas donner à autrui des secrets ou des informations qui pourraient être utilisées contre les intérêts de l'entreprise. Selon la jurisprudence, le conseiller doit, à titre individuel, prendre une décision bien réfléchie ${ }^{12}$ qui tienne compte tant des intérêts de l'entreprise que de ceux des travailleurs, qui ont par exemple besoin de savoir s'il existe un plan de restructuration.

Il est évident que le destinataire des informations n'est pas nécessairement capable de les comprendre. Un bilan peut être très compliqué et occulter des points sensibles. Un " document stratégique " présenté par la direction peut utiliser une langue très diplomatique qui n'est pas celle des travailleurs. A titre individuel, un membre du conseil n'a pas le droit de consulter un expert rémunéré par l'entreprise. Pour recourir à l'aide d'un expert, il faut une décision du conseil, qui dépend des voix du capital. En principe, les dirigeants de l'entreprise ont eux-mêmes une expérience très longue dans les affaires et une connaissance approfondie du marché. De plus, ils disposent de collaborateurs qualifiés dans de nombreux domaines. Un représentant des travailleurs n'a pas de tels moyens à sa disposition. Il n'a pas le temps de se former car il doit remplir sa tâche quotidienne dans l'entreprise ou dans le syndicat. Pour le dire de manière imagée, une discussion sur la politique de l'entreprise ressemble à un match de football entre le Paris St. Germain et une équipe d'amateurs. Ces derniers peuvent, par hasard, marquer un but, mais le résultat final restera inchangé.

11 Voir l'expertise de Spindler, https://www.boeckler.de/1194 1273.htm (3.4.2018).

12 Voir les références dans Oetker in Müller-Glöge/Preis/Schmidt (dir.), Erfurter Kommentar zum Arbeitsrecht, 18. Aufl. 2018, § 116 AktG Rn. 6. 
Même partielles, les informations peuvent s'avérer importantes pour le fonctionnement du conseil d'établissement. II peut ainsi consolider ou améliorer sa position quand il sait quelle est l'intention de la direction sur telle ou telle question. Transmettre les informations au conseil d'établissement représente pourtant un problème juridique car la direction peut soutenir que l'obligation de discrétion n'a pas été observée. En pratique, les représentants des travailleurs sont souvent en même temps membres du conseil d'établissement si bien que le "transfert » ne se heurte pas à de grandes difficultés psychologiques: c'est une moitié du cerveau qui informe l'autre.

Les représentants des travailleurs au sein du conseil de surveillance reçoivent la même somme d'argent que les représentants du capital. Cela peut être un montant modeste de 3000,00 euros par an dans une entreprise relativement petite, mais cela peut être aussi une somme considérable au sein des grandes banques ou, par exemple, chez Siemens et BASF allant jusqu'à 200000 euros par an. Les représentants qui sont membres d'un syndicat promettent par écrit avant l'élection de reverser cette rémunération (sauf une somme de 5000 euros) à la fondation "Hans Böckler » qui est gérée par les grands syndicats majoritaires du DGB. Les membres proposés par un syndicat le font effectivement. Pour ce qui est des autres, "une règle connaît de nombreuses exceptions en fait ». La sanction de ne plus être candidat aux prochaines élections ne fonctionne pas toujours car les travailleurs continuent à donner un soutien à ces personnes et l'autorité syndicale est très limitée.

La fondation «Hans Böckler » utilise l'argent pour financer des recherches scientifiques dans l'intérêt de travailleurs qui se révèlent fort utiles pour les syndicats: S'il y a des négociations collectives dans une branche, les spécialistes de la fondation Böckler savent très bien quelle est la situation économique des entreprises. De plus, la fondation finance parfois des recherches empiriques sur la dissonance entre la réalité et le droit. La fondation offre aussi des séminaires concernant la politique d'une entreprise spécifique, mais cela ne peut changer le déséquilibre entre capital et travail qui existe dans les délibérations du conseil de surveillance.

\section{B - Cogestion et Sociétés multinationales}

Les trois modèles de cogestion s'appliquent uniquement aux sociétés qui ont leur siège social en Allemagne. Les filiales domiciliées dans un autre État-Membre de I'Union européenne ne sont pas incluses. L'argument voulant qu'il s'agisse d'une discrimination indirecte en raison de la nationalité a été rejeté par la Cour de Justice de Luxembourg : étant donné que la question de la participation des travailleurs n'a été jamais réglée par I'Union Européenne, ce problème se trouve en dehors du champ d'application du droit de I'Union ${ }^{13}$.

Si une entreprise étrangère a des filiales en Allemagne, la cogestion ne s'applique pas. Si elle a une filiale employant un nombre suffisant de travailleurs, les règles de cogestion s'appliquent mais le directoire d'une telle entreprise et surtout le conseil de surveillance ne peuvent pas prendre de véritables décisions. La société mère arrête dès lors toutes les décisions qui sont d'une certaine importance. 
Le droit de l'Union européenne donne aux entreprises le droit de s'installer formellement dans un pays et de poursuivre toutes les activités dans un autre État-Membre. Si une telle société (exemple traditionnel : une «Limited » britannique) déploie 100\% de ses activités en Allemagne, les lois sur la cogestion ne s'appliquent pas parce qu'il ne s'agit pas d'une société par actions ou d'une Gmbh ou d'une autre forme d'entreprise allemande régie par le droit allemand. Les syndicats revendiquent l'inclusion de ces entreprises dans le système de cogestion tandis que le législateur allemand demeure très hésitant à cet égard.

\section{C - Des VOIES POUR ÉVITER LA COGESTION}

Malgré la portée très limitée de la cogestion, certaines entreprises ne souhaitent pas avoir des représentants des travailleurs au sein de leur conseil de surveillance. Elles ont plusieurs moyens à leur disposition, en principe considérés comme légaux.

- Les propriétaires de l'entreprise ont le pouvoir de changer de forme juridique. Une société à responsabilité limitée est ainsi transformée en une société en commandite simple qui ne relève pas du régime de la cogestion. Cette voie n'est pratiquement jamais empruntée car elle implique la responsabilité personnelle des propriétaires.

- Une autre forme juridique semble plus attrayante : une combinaison entre AG ou $\mathrm{GmbH}$ et une société en commandite simple. Dans cette hypothèse, la société par action ou GmbH prend la position de l'associé personnellement responsable et les autres propriétaires prennent le rôle des commanditaires. Dans le même temps, ils sont les actionnaires de la société par actions ou les associés de la $\mathrm{GmbH}$. Depuis l'époque de Weimar, cette forme de société a été reconnue par la jurisprudence. Dans le «Mitbestimmungsgesetz » de 1976, le législateur a pris en considération cette forme spécifique de société. Les salariés de la société en commandite simple sont traités comme des salariés de la société par actions ou de la Gmbh. Si au total, ils comptent plus de 2000 salariés, la société par action ou la $\mathrm{GmbH}$ sera sous le régime de la cogestion. Mais le contrat de société peut réserver une position relativement modeste à la société par action ou à la $\mathrm{GmbH}$ si bien que l'influence des organes « cogérés » restera très limitée. En plus, la pratique offre une autre piste : les propriétaires peuvent former deux AG ou deux $\mathrm{GmbH}$, chacune employant moins de 2000 salariés. Alors les deux sociétés s'associent et forment une société en nom collectif, la règle exigeant d'additionner le nombre des salariés ne s'applique pas dans un tel cas.

- Les associés peuvent procéder à une restructuration pour ne plus atteindre le seuil de 2000 salariés. Par exemple, l'entreprise allemande se sépare formellement d'un département qui sera dirigé par une société domiciliée à l'étranger. Comme les sociétés à l'étranger restent hors du champ d'application des lois de cogestion, le nombre des travailleurs en Allemagne n'atteint plus le nombre requis de 2000 , ou de 500, suivant le cas.

- Le droit de l'Union européenne offre des voies beaucoup plus confortables pour les employeurs. Les associés peuvent former une AG ou une GmbH conformément au droit d'un autre État-Membre qui ne connaît pas la cogestion. Par la suite, un contrat est conclu entre cette société et la société domiciliée en Allemagne. Cette dernière se soumet alors aux directives de la société «étrangère ». Les associés sont identiques (ou presque). Un tel contrat est admis par la jurisprudence allemande. Le conseil de surveillance de la société allemande continue d'exister mais on discute uniquement de la mise en œuvre de décisions qui sont prises ailleurs. 
Sauf dans de grandes sociétés par actions dont les propriétaires sont souvent inconnus, la cogestion dépend de la bonne volonté des propriétaires. C'est une raison de plus pour les représentants des travailleurs de ne pas perturber la bonne atmosphère des discussions et d'éviter dans la mesure du possible des questions désagréables pour le capital et surtout des actions collectives. II en va différemment dans les grandes firmes telles Siemens ou Opel au sein desquelles la cogestion n'est pas menacée. Si de sérieux problèmes surgissent pour les travailleurs (comme dans le cas d'Opel), leur voix est écoutée partout.

\section{D - AUtRES FORMES DE COGESTION?}

Le déplacement de l'entreprise à l'étranger et la fermeture d'une usine ne peuvent pas être empêchés par un vote du conseil de surveillance, faute de majorité des travailleurs. Y a-t-il au moins une possibilité d'en faire un objet de négociation collective?

La doctrine allemande est divisée à ce sujet. Un courant soutient que les " décisions des entrepreneurs " (Unternehmerentscheidungen) doivent rester libres. Seraient dès lors illégales les stipulations d'une convention collective qui auraient pour objet d'interdire une décision ou de restreindre la liberté de l'entreprise en imposant des conditions. Un autre courant considère qu'il n'y a aucune base juridique susceptible de donner une telle protection aux décisions unilatérales des entrepreneurs. Conclure une convention collective portant sur de telles décisions serait au contraire conforme au principe de l'autonomie collective. Pour cette partie de la doctrine allemande, la conclusion d'une convention collective est une forme d'exercice de la liberté de l'entrepreneur ${ }^{14}$.

La jurisprudence est en faveur de la deuxième position, sans toutefois l'exprimer de manière explicite. Au cours des vingt dernières années, on relève plusieurs cas dans lequels les employeurs ont voulu réduire les salaires fixés par des conventions collectives de branche. Les syndicats ont accepté une telle concession seulement si les représentants des travailleurs disposaient d'un véritable contrôle sur les affaires de l'entreprise. Dans certaines conventions collectives, on a créé des commissions paritaires qui devaient donner leur accord sur toutes les décisions majeures de l'entreprise. Dans d'autres conventions il y avait une obligation de créer de nouveaux postes ou de sauvegarder ceux qui existaient déjà. La Cour fédérale du Travail a accepté toutes ces conventions sans remettre en question leur validité ${ }^{15}$. La particularité de ces conventions est qu'elles ont été conclues d'une façon volontaire, sans menace de grève.

14 Pour plus de détails, W. Däubler, Die Unternehmerfreiheit im Arbeitsrecht - eine unantasstbare Größe? 2012 et W. Däubler, Tarifverträge zur Unternehmenspolitik? Rechtliche Zulässigkeit und faktische Bedeutung, 2016 (http://www.hugo-sinzheimer-institut.de/fileadmin/user data hsi/ Veroeffentlichungen/HSI Schriftenreihe/Wolfgang Daeubler.pdf et http://www.hugo-sinzheimerinstitut.de/fileadmin/user data hsi/Veroeffentlichungen/HSI Schriftenreihe/Daeubler Tarifvertraege zur Unternehmenspolitik.pdf ).

15 BAG 24.4.2007 - 1 AZR 252/06 - AP Nr. 2 zu § 1 RVG Sozialplan; NAG 11.9.1991 - 4 AZR 71/91 NZA 1992, 321; BAG 26.1.2011 - 4 AZR 159/09 - NZA 2011, 808; LAG Niedersachsen 18.5.2011 - 17 SaGa 1939/10 - AiB 2011, 481. 


\section{SYSTİ̀ME ALLEMAND DE PARTICIPATION DES TRAVAILLEURS}

Dans deux cas particuliers (Otis et Heidelberger Druckmaschinen), les travailleurs ont fait grève face à une fermeture imminente de l'établissement. Les revendications ne consistaient pas à demander la sauvegarde de tous les postes de travail, le syndicat craignant que la grève soit déclarée illégale par les tribunaux de travail. Les revendications se sont donc limitées aux conséquences « sociales » de la fermeture : délais de préavis entre un et deux ans, rémunération normale pour trois ans après la fin du contrat de travail pour être capable de se former à un autre emploi. Si ces revendications avaient été acceptées, elles auraient réduit à néant l'effet économique de la fermeture. La Cour fédérale a décidé qu'il n'y avait aucun contrôle judiciaire à exercer sur la proportionnalité des revendications. L'influence que de telles revendications peuvent avoir sur la décision de fermeture par ailleurs justifiée ne constitue pas un obstacle à la grève ${ }^{16}$. D'une façon expresse, la Cour a laissé de côté la légalité d'une revendication de sauvegarder les postes de travail existants en pratique il n'y a aucune nécessité de s'aventurer sur ce terrain dangereux. De ce point de vue et pour conclure, les négociations collectives assurent aux travailleurs plus de succès que la cogestion.

16 BAG 24.4.2007 - 1 AZR 252/06 - NZA 2007, 987; voir aussi LAG Niedersachsen 2.6.2004 - 7 Sa 819/04 - AP Nr. 164 zu Art. 9 GG Arbeitskampf.

\section{WOLFGANG DÄUBLER}

Professeur à l'Université de Brême.

Thèmes de recherche : La digitalisation et les relations de travail, les effets de la concurrence internationale sur les travailleurs.

\section{Publications :}

«Challenges to Labour Law», in Perulli (ed.), L'Idea di Diritto del Lavoro, Oggi. In ricordo di Giorgio Ghezzi, Wolters Kluwer Italia, 2016, p. 485-506.

«Crowdwork: Datore di lavoro in fuga?», Giornale di diritto del lavoro e di relazioni industriali, $n^{\circ} 151,2016$, p. 3. 